\title{
О РАСШИРЕНИИ ХЬЮИТТА И $\tau$-РАСПОЛОЖЕННОСТИ ФУНКЦИОНАЛЬНЫХ ПРОСТРАНСТВ
}

\author{
А. В. Осипов
}

\begin{abstract}
В работе исследуется связь между расширениями типа расширений Хьюитта и пространствами строго $\tau-F$-отображений. Получен критерий вещественной полноты пространства бэровских отображений класса $\alpha$. Доказано, что пространство $B(X, G)$ бэровских отображений $G$ - $z$-нормального пространства $X$ в не компактное метризуемое сепарабельное пространство $G$ является линделёфовым тогда и только тогда, когда $X$ счетно.
\end{abstract}

Ключевые слова: вещественно полные пространства, слабая функциональная теснота, бэровское отображение, $\tau$-расположенность, расширение Хьюитта.

\section{A. V. Osipov. On the Hewitt realcompactification and $\tau$-placedness of function spaces.}

We study the relation between extensions of the Hewitt realcompactification type and spaces of strictly $\tau$ - $F$ functions. A criterion is obtained for the realcompleteness of the space of Baire functions of class $\alpha$. It is proved that the space $B(X, G)$ of Baire functions from a $G$ - $z$-normal space $X$ to a noncompact metrizable separable space $G$ is Lindelöf if and only if $X$ is countable.

Keywords: realcomplete spaces, weak functional tightness, Baire function, $\tau$-placedness, Hewitt realcompactification.

MSC: 54C35 54C25

DOI: $10.21538 / 0134-4889-2019-25-4-177-183$

\section{Введение}

Один из важных видов топологической полноты был введен Э. Хьюиттом и Л. Нахбином в [1]. Пространство $X$ называется вещественно полным, если оно гомеоморфно замкнутому подпространству пространства $\mathbb{R}^{\tau}$ для некоторого кардинального числа $\tau$. В литературе вещественно полные пространства иногда называют $Q$-пространствами, или вещественно компактными пространствами (realcompact space), или $R$-полными пространствами, или пространствами Хьюитта - Нахбина [1-4].

Полным по Дьедонне называют пространство, полное относительно максимальной равномерной структуры, совместимой с его топологией. Полные по Дьедонне пространства характеризуются как гомеоморфные образы замкнутых подпространств произведений метрических пространств [3].

Кардинал $\tau$ называют измеримым по Уламу, если на множестве мощности $\tau$ существует максимальная центрированная система с пустым пересечением, пересечение любого счетного семейства которой не пусто.

В системе $Z F C$ (Цермело - Френкеля) аксиом теории множеств можно считать, что измеримых кардиналов не существует. При этом предположении каждое полное по Дьедонне пространство вещественно полно. Обратное верно всегда.

Заметим, что пространство $C_{p}(X)$ непрерывных вещественнозначных функций, определенных на тихоновском пространстве $X$, с топологией поточечной сходимости вещественно полно в том и только том случае, если $C_{p}(X)$ полно по Дьедонне. Это следует из того, что число Суслина пространства $C_{p}(X)$ счетно $[2 ; 5]$. 
В работах [5-8] были исследованы связи между расширениями типа Хьюитта и пространствами строго $\tau$-непрерывных функций.

Отображение $f: X \rightarrow Y$ называется строго $\tau$-непрерывным, если для каждого множества $A \subset X$ такого, что $|A| \leq \tau$, найдется непрерывное отображение $g: X \rightarrow Y$, для которого $g|A=f| A$ (т. е. $f(x)=g(x)$ при всех $x \in A$ ) [5]. При $Y=\mathbb{R}$ пространство всех строго $\tau$-непрерывных функций на пространстве $X$ с топологией поточечной сходимости обозначают через ${ }^{(\tau)} C_{p}(X)[8]$.

О п р е д е л е н и е 1 . Слабой функциональной теснотой $t_{Y}(X)$ пространства $X$ будем называть наименьший бесконечный кардинал $\tau$ такой, что каждое строго $\tau$-непрерывное отображение $f: X \rightarrow Y$ непрерывно.

При $Y=\mathbb{R}$ получаем слабую функциональную тесноту $t_{\mathbb{R}}(X)$ пространства $X$ [5].

Напомним, что $A \subset X$ есть множество типа $G_{\tau}$ в $X$, если существует семейство $\gamma$ открытых в $X$ множеств такое, что $A=\bigcap \gamma$ и $|\gamma| \leq \tau$.

Множество $A \subset X$ называют $\tau$-расположенным в $X$, если для каждой точки $x \in X \backslash A$ найдется множество $P$ типа $G_{\tau}$ в $X$ такое, что $x \in P \subset X \backslash A$.

Числом Хьюитта - Нахбина пространства $X$ называют кардинал $q(X)=\min \{\tau: X \tau$ расположено в $\beta X\}+\aleph_{0}$. Известно, что $q(X)=\aleph_{0}$ в том и только том случае, если пространство $X$ вещественно полно [5].

А. В. Архангельский доказал, что для любого тихоновского пространства $X$ выполняется равенство $t_{\mathbb{R}}(X)=q\left(C_{p}(X)\right)$ [5, теорема II.4.16].

В работе [8] О.Г. Окунев определяет для каждых топологического пространства $X$ и кардинального числа $\tau$ расширение, обобщающее понятие расширения Хьюитта $\nu X$.

О п р е д е л е н и е 2. $\tau$-расширением Хъюитта пространства $X$ называется пространство $\nu_{\tau} X=\left\{x \in \beta X\right.$ : каждое множество типа $G_{\tau}$ в $\beta X$, которое содержит точку $x$, имеет непустое пересечение с $X\}$ в топологии подпространства $\beta X$.

Очевидно, что $\nu_{\aleph_{0}} X$ совпадает с расширением Хьюитта $\nu X$ и $X \subseteq \nu_{\tau} X \subseteq \nu_{\lambda} X$ при $\lambda \leq \tau$. Ясно также, что $\nu_{\tau} X=X$, если $q(X) \leq \tau$.

О.Г. Окунев доказал, что для тихоновского пространства $X$ пространства ${ }^{(\tau)} C_{p}(X)$ и $\nu_{\tau} C_{p}(X)$ гомеоморфны [8, теорема 2].

В этой работе мы исследуем свойство $\tau$-расположенности и число Хьюитта - Нахбина для широкого класса пространств отображений, включающего пространства $B_{\alpha}(X, Y)$ бэровских отображений класса $\alpha \in\left[0, \omega_{1}\right]$. В частности, мы получим критерий вещественной полноты и свойства линделёфовости для пространства $B(X)$ бэровских функций, определенных на тихоновском пространстве $X$.

Основные определения и обозначения. Все рассматриваемые в этой работе топологические пространства являются бесконечными и тихоновскими. Все пространства отображений подразумеваются наделенными топологией поточечной сходимости.

Для топологических пространств $X$ и $Y$ через $Y^{X}$ будем обозначать множество $\{f: f$ : $X \rightarrow Y\}$ всех отображений пространства $X$ в пространство $Y$.

О п р е д е л е н и е 3 . Пусть $X$ и $Y$ - топологические пространства и $F(X, Y) \subseteq Y^{X}$. Отображение $f: X \rightarrow Y$ будем называть строго $\tau$-F-отображением, если для каждого множества $A \subset X$ такого, что $|A| \leq \tau$, найдется отображение $g \in F(X, Y)$, для которого $g|A=f| A$.

Множество всех строго $\tau$-F-отображений в пространстве $Y^{X}$ будем обозначать через ${ }^{(\tau)} F(X, Y)$.

О п р е д е л е н и е 4. Пусть $X$ и $Y$ - топологические пространства и $F(X, Y) \subseteq Y^{X}$. Слабой функииональной теснотой $t_{Y, F}(X)$ пространства $X$ будем называть наименьший бесконечный кардинал $\tau$ такой, что каждое строго $\tau$-F-отображение $f$ принадлежит $F(X, Y)$.

Основные обозначения можно найти в работах $[3 ; 9 ; 10]$. 


\section{1. Основные результаты}

Лемма 1.1. Пусть $X-$ топологическое пространство, $Y$ - топологическое пространство с псевдохарактером, равным $\tau$, и $F(X, Y) \subseteq Y^{X}$. Eсли $t_{Y, F}(X) \leq \tau$, то пространство $F(X, Y) \tau$-расположено в $Y^{X}$.

Д о к а з а т е л ь с т в о. Пусть $g \in Y^{X} \backslash F(X, Y)$. Из условия $t_{Y, F}(X) \leq \tau$ следует, что существует $A \subset X$ такое, что $|A| \leq \tau$ и $g|A \neq f| A$ при всех $f \in F(X, Y)$. Рассмотрим отображение сужения $\pi: Y^{X} \rightarrow Y^{A}$, т. е. $\pi(h)=h \mid A$ для всех $h \in Y^{X}$.

Множество $Z=\pi(F(X, Y)) \tau$-расположено в $Y^{A}$, так как $|A| \leq \tau$ и $Y$ имеет псевдохарактер, равный $\tau$ (все одноточечные подмножества $Y^{A}$ имеют тип $G_{\tau}$ ). Так как $\pi(g)=g \mid A \notin Z$ и $\{\pi(g)\}$ - множество типа $G_{\tau}$ в $Y^{A}$, то $P=\pi^{-1}(\pi(g))$ - множество типа $G_{\tau}$ в $Y^{X}$ и $g \in P \subset$ $Y^{X} \backslash F(X, Y)$.

Лемма доказана.

Лемма 1.2. Пусть $X$ и $Y-$ топологические пространства и $F(X, Y) \subseteq Y^{X}$. Eсли $F(X, Y)$ $\tau$-расположено в $Y^{X}, \operatorname{mot}_{Y, F}(X) \leq \tau$.

Д ок а з а т е л ь с т в о. Пусть $g \in{ }^{(\tau)} F(X, Y)$ и $P$ - любое множество типа $G_{\tau}$ в $Y^{X}$ такое, что $g \in P$. Пусть $P=\bigcap\left\{W_{\alpha}: \alpha<\tau\right\}$, где $W_{\alpha}$ - открытое множество в $Y^{X}$ для каждого $\alpha<\tau$. Так как $g \in W_{\alpha}$, то для каждого $\alpha<\tau$ существует базисная окрестность $S_{\alpha}$ точки $g$ такая, что $g \in S_{\alpha} \subseteq W_{\alpha}$. Здесь

$$
\begin{gathered}
S_{\alpha}=\left\langle g, x_{1, \alpha}, \ldots, x_{n(\alpha), \alpha}, V_{1, \alpha}, \ldots, V_{n(\alpha), \alpha}\right\rangle:= \\
\left\{f \in Y^{X}: f\left(x_{i, \alpha}\right) \in V_{i, \alpha} \text { для каждого } i=1, \ldots, n(\alpha)\right\},
\end{gathered}
$$

где $x_{i, \alpha} \in X$ и $V_{i, \alpha}$ - открытое множество в пространстве $Y$ такое, что $g\left(x_{i, \alpha}\right) \in V_{i, \alpha}$ для $i=1, \ldots, n(\alpha)$ и $n(\alpha) \in \mathbb{N}$. Очевидно, что $g \in \bigcap_{\alpha<\tau} S_{\alpha} \subseteq P$.

Пусть $A=\bigcup_{\alpha<\tau}\left\{x_{1, \alpha}, \ldots, x_{n(\alpha), \alpha}\right\}$. Тогда $|A| \leq \tau$ и $g \in\left\{f \in Y^{X}: f|A=g| A\right\} \subset P$.

Так как $g \in{ }^{(\tau)} F(X, Y)$, существует $h \in F(X, Y)$ такое, что $h|A=g| A$. Тогда $h \in P$. Итак, $P \cap F(X, Y) \neq \varnothing$ для любого множества $P$ типа $G_{\tau}$ в $Y^{X}$, содержащего $g$. Так как $F(X, Y)$ $\tau$-расположено в $Y^{X}$, заключаем, что $g \in F(X, Y)$. Следовательно, $t_{Y, F}(X) \leq \tau$.

Лемма доказана.

Теорема 1.1. Для топологического пространства $X$, вещественно полного пространства $Y$ со счетным характером и плотного подмножества $F(X, Y) \subseteq Y^{X}$ выполняется равенство $q(F(X, Y))=t_{Y, F}(X)$.

Д о к а з а т е л ь с т в о. Положим $\tau=t_{Y, F}(X)$. По лемме 1.1 пространство $F(X, Y) \tau$ расположено в $Y^{X}$. Произведение любого числа вещественно полных пространств является вещественно полным [3, теорема 3.11.5]. Следовательно, вещественно полное пространство $Y^{X} \aleph_{0}$-расположено в $\beta\left(Y^{X}\right)[2]$. Пространство $F(X, Y) \tau$-расположено в $\beta\left(Y^{X}\right)[5$, предложение 4.9]. Значит, $q(F(X, Y)) \leq t_{Y, F}(X)$.

Положим $\lambda=q(F(X, Y))$. Произведение любого числа пространств счетного характера является московским пространством, т.е. любое канонически замкнутое множество представимо в виде объединения $G_{\delta}$-множеств [10, следствие 6.3.15]. Следовательно, $Y^{X}$ - московское пространство и $F(X, Y) \lambda$-расположено в $Y^{X}$ [5, предложение II.4.13]. Применяя лемму 1.2, заключаем, что $t_{Y, F}(X) \leq \lambda$.

Таким образом, $t_{Y, F}(X) \leq q(F(X, Y))$ и, значит, $q(F(X, Y))=t_{Y, F}(X)$.

Теорема доказана. 
Следствие 1.1. Для топологического пространства $X$, вещественно полного пространства $Y$ со счетным характером и плотного подмножества $F(X, Y) \subseteq Y^{X}$ слабал функциональная теснота $t_{Y, F}(X)$ пространства $X$ счетна в том и только том случае, когда пространство $F(X, Y)$ вещественно полно.

Следующая теорема является обобщением теоремы Окунева, но по сути использует идею доказательства теоремы 2 из работы [8] для функционального пространства $C_{p}(X)$.

Теорема 1.2. Для топологического пространства $X$, топологического пространства $Y$ с весом не больше, чем $\tau$ и плотного подмножества $F(X, Y) \subseteq Y^{X}$ пространства $^{(\tau)} F(X, Y)$ и $\nu_{\tau} F(X, Y)$ гомеоморфны.

Д о к а з а т е л ь с т в о. Заметим, что

$$
{ }^{(\tau)} F(X, Y)=\bigcap\left\{\pi_{A}(F(X, Y)) \times Y^{X \backslash A}: A \subset X,|A| \leq \tau\right\},
$$

где $\pi_{A}: Y^{X} \rightarrow Y^{A}-$ проекция для каждого $A \subset X$. Заметим, что $w\left(\pi_{A}(F(X, Y)) \leq \tau\right.$, $q\left(\pi_{A}(F(X, Y)) \times Y^{X \backslash A}\right) \leq \tau$ и $q\left({ }^{(\tau)} F(X, Y)\right) \leq \tau$. Осталось доказать, что $F(X, Y) C$-вложено в ${ }^{(\tau)} F(X, Y)$. Пусть $g: F(X, Y) \rightarrow \mathbb{R}-$ непрерывная функция. Так как $F(X, Y)$ - плотное подмножество в $Y^{X}$ и $Y$ - пространство с весом, не превосходящем $\tau$, мы можем применить факторизационную теорему [10, следствие 1.7.4]. Тогда найдутся множество $A \subset X$ с $|A| \leq \tau$ и непрерывная функция $\varphi: \pi_{A}(F(X, Y)) \rightarrow \mathbb{R}$ такие, что $g=\varphi \pi_{A}$. Очевидно, что отображение $p=\varphi \pi_{A}: \pi_{A}(F(X, Y)) \times Y^{X \backslash A} \rightarrow \mathbb{R}$ непрерывно, и так как ${ }^{(\tau)} F(X, Y) \subset \pi_{A}(F(X, Y)) \times Y^{X \backslash A}$, то требуемое продолжение функции $g$ построено.

Теорема доказана.

\section{2. Приложение для бэровских отображений}

Для топологических пространств $X$ и $Y$ определим классы бэровских отображений. Пусть $B_{0}(X, Y):=C(X, Y)$ - множество непрерывных отображений пространства $X$ в $Y$. Множество $B_{\alpha}(X, Y)$ состоит из всех поточечных пределов сходящихся в пространстве $Y^{X}$ последовательностей отображений классов $<\alpha . B(X, Y):=B_{\omega_{1}}(X, Y)=\bigcup_{\alpha<\omega_{1}} B_{\alpha}(X, Y)$ - пространство бэровских отображений. Если $Y=\mathbb{R}$, то $B_{\alpha}(X):=B_{\alpha}(X, \mathbb{R})$ и $B(X):=B_{\omega_{1}}(X, \mathbb{R})$.

Для пространства $B_{\alpha}(X, Y)$ бэровских отображений класса $\alpha$ определение строго $\tau$ - $B_{\alpha}$-отображения имеет следующий вид.

О п р е д е л е н и е 5 . Отображение $f: X \rightarrow Y$ будем называть строго $\tau$-бэровским класса $\alpha$, если для каждого множества $A \subset X$ такого, что $|A| \leq \tau$, найдется отображение $g: X \rightarrow Y$ бэровского класса $\alpha$, для которого $g|A=f| A$.

О п р е д е л е н и е 6 . Слабой $B_{\alpha}$-теснотой $t_{Y, B_{\alpha}}(X)$ пространства $X$ будем называть наименьший бесконечный кардинал $\tau$ такой, что каждое строго $\tau$-бэровское отображение $f$ : $X \rightarrow Y$ класса $\alpha$ является бэровским отображением класса $\alpha$, т. е. $f \in B_{\alpha}(X, Y)$.

Следствием теоремы 1.1 является следующее утверждение.

Теорема 2.1. Для топологического пространства $X$, вещественно полного пространства $Y$ со счетным характером и $\alpha \in\left[0, \omega_{1}\right]$ выполняется равенство $q\left(B_{\alpha}(X, Y)\right)=t_{Y, B_{\alpha}}(X)$.

Следствие 2.1. Для топологического пространства $X$, вещественно полного пространства $Y$ со счетным характером и $\alpha \in\left[0, \omega_{1}\right]$ слабал $B_{\alpha}$-теснота $t_{Y, B_{\alpha}}(X)$ пространства $X$ счетна в том и только том случае, когда пространство $B_{\alpha}(X, Y)$ вещественно полно.

В частности, при $Y=\mathbb{R}$ получаем такое следствие теоремы 2.1. 
Следствие 2.2. Для топологического пространства $X u \alpha \in\left[0, \omega_{1}\right]$ выполняется равенство $q\left(B_{\alpha}(X)\right)=t_{B_{\alpha}}(X)$.

Следствием теоремы 1.2 для бэровских отображений является следующее утверждение.

Теорема 2.2. Для топологического пространства $X$, топологического пространства $Y$ с весом не более чем $\tau$ u $\alpha \in\left[0, \omega_{1}\right]$ пространства ${ }^{(\tau)} B_{\alpha}(X, Y)$ и $\nu_{\tau} B_{\alpha}(X, Y)$ гомеоморфны.

Следствие 2.3. Для топологического пространства $X, \alpha \in\left[0, \omega_{1}\right] u \tau \geq \aleph_{0}$ пространства $^{(\tau)} B_{\alpha}(X)$ и $\nu_{\tau} B_{\alpha}(X)$ гомеоморфны.

О п р е д е л е н и е 7 . Пусть $X$ и $Y$ - топологические пространства. Пространство $X$ будем называть $Y$-z-нормалъным, если $X-T_{1}$-пространство и для любых дизъюнктного семейства $F_{1}, \ldots, F_{k}$ нуль-множеств пространства $X$ и точек $y_{1}, \ldots, y_{k}$ пространства $Y$ существует непрерывное отображение $f: X \rightarrow Y$ такое, что $f\left(F_{i}\right)=y_{i}$ для каждого $i=1, \ldots, k$.

Лемма 2.1. Пусть $X$ является $Y$-z-нормальным для некоторого топологического пространства $Y, F-$ нуль-множество пространства $X$ и $a, b \in Y$. Тогда отображение $f$ : $X \rightarrow Y$ mакое, что $f(F)=\{a\}$ u $f(X \backslash F)=\{b\}$, является бэровским отображением первого класса.

Д о к а з а т е л ь с т в о. Так как $F$ - нуль-множество пространства $X$, множество $X \backslash F$ функционально открыто в $X$ и $X \backslash F=\bigcup_{j=1}^{\infty} S_{j}$, где $S_{j}$ - нуль-множества пространства $X$ и $S_{j} \subset S_{j+1}$ для $j \in \omega$. Так как $X-Y$-z-нормальное пространство, для любого $j \in \omega$ существует $f_{j} \in C_{p}(X, Y)$ такое, что $f_{j}(F)=\{a\}$ и $f_{j}\left(S_{j}\right)=\{b\}$. Осталось заметить, что отображение $f=\lim _{j \rightarrow \infty} f_{j}$ является бэровским отображением первого класса и $f(F)=\{a\}, f(X \backslash F)=\{b\}$.

Лемма доказана.

Теорема 2.3. Пусть $Y$ - топологическое пространство счетного псевдохарактера и $X-Y$-z-нормальное пространство. Тогда ${ }^{\left(\aleph_{0}\right)} B_{2}(X, Y)=Y^{X}$.

Д ок а з а т е ль с т в о. Пусть $f \in Y^{X}, A=\left\{a_{i}: i \in \omega\right\} \subset X$ и $y \in Y$. Для каждой точки $a \in A$ зафиксируем множество $Z_{a}:=f^{-1}(f(a))$. Так как $Y$ - тихоновское пространство со счетным псевдохарактером, множество $\{f(a)\}$ - нуль-множество в пространстве $Y$. Следовательно, $Z_{a}$ - нуль-множество в пространстве $X$.

Рассмотрим отображения $f_{n}: X \rightarrow Y$ такие, что $f_{n} \mid Z_{a}=f(a)$ и

$$
f_{n}\left(X \backslash \bigcup_{a \in\left\{a_{1}, \ldots, a_{n}\right\}} Z_{a}\right) \subseteq\{y\} \text { для всех } a \in\left\{a_{1}, \ldots, a_{n}\right\} \text { и каждого } n \in \omega .
$$

По лемме 2.1 отображение $f_{n} \in B_{1}(X, Y)$ для каждого $n \in \omega$. Рассмотрим отображение $g:=$ $\lim f_{n}$ при $n \rightarrow \infty$. Отображение $g$ определено во всех точках $X$, и, значит, $g \in B_{2}(X, Y)$. В силу произвольности отображения $f$ и множества $A$ получаем, что $Y^{X} \subseteq{ }^{\left(\aleph_{0}\right)} B_{2}(X, Y)$.

Теорема доказана.

Следствие 2.4. Пусть $X$ - тихоновское пространство. Тогда ${ }^{\left(\aleph_{0}\right)} B_{2}(X)=\mathbb{R}^{X}$.

Таким образом, вещественная полнота пространства $B_{\alpha}(X)(\alpha \geq 2)$ влечет (теоремы 1.1 и $1.2)$ вырожденность пространства $X$, выражающуюся в равенстве $B_{\alpha}(X)=\mathbb{R}^{X}$.

Напомним, что топологическое пространство $X$ называется линделёбовым, если из любого открытого покрытия пространства $X$ можно выделить счетное подпокрытие. Хорошо известно, что любое линделёфовое пространство вещественно полно [3, теорема 3.11.12] и нормально [3, теорема 3.8.2]. 
Теорема 2.4. Пусть $X-G$-z-нормальное пространство, где $G$ - некомпактное топологическое пространство со счетной базой и $\alpha \in\left[2, \omega_{1}\right]$. Тогда линделёбовость пространства $B_{\alpha}(X, G)$ эквивалентна счетности пространства $X$.

Д о к а з а т е л ь с т в о. Предположим, что $B_{\alpha}(X, G)$ - линделёфово пространство. Тогда $B_{\alpha}(X, G)$ вещественно полно и по теореме $2.3 B_{\alpha}(X, G)=G^{X}$. Отсюда следует, что пространство $G^{X}$ нормально. Так как $G$ - некомпактное пространство, то $G^{X}-$ нормальное пространство тогда и только тогда, когда $X$ счетно [11].

Если $X$ счетно, то $G^{X}$ - пространство со счетной базой (сепарабельное метризуемое), а значит, наследственно линделёфово. Следовательно, пространство $B_{\alpha}(X, G)$ линделёфово.

Теорема доказана.

В качестве следствия получаем результат, доказанный А. В. Пестряковым в работе [12].

Следствие 2.5. Пусть $X-$ тихоновское пространство и $\alpha \in\left[2, \omega_{1}\right]$. Тогда линделёбовость пространства $B_{\alpha}(X)$ эквивалентна счетности пространства $X$.

В частности, линделёфовость пространства бэровских функций на тихоновском пространстве $X$ эквивалентна счетности пространства $X$.

В о п р о с 1. Существует ли некомпактное топологическое пространство со счетной базой $G$ и несчетное $G$ - $z$-нормальное пространство $X$ такие, что $B_{1}(X, G)$ линделёфово?

Ослаблением вопроса 1 может быть следующий вопрос.

В о п р о с 2. Существует ли некомпактное топологическое пространство со счетной базой $G$ и несчетное $G$ - $z$-нормальное пространство $X$ такие, что $B_{1}(X, G)$ нормально и вещественно полно?

\section{СПИСОК ЛИТЕРАТУРЫ}

1. Hewitt E. Rings of real-valued continuous functions, I // Trans. Amer. Math. Soc. 1948. Vol. 64, no. 1. P. $45-99$.

2. Архангельский А.В., Пономарев В.И. Основы общей топологии в задачах и упражнениях. М.: Наука, 1974. 424 с.

3. Энгелькинг Р. Общая топология. М.: Мир, 1986. 752 с.

4. Nachbin L. Topological vector spaces of continuous functions // Proc. Nat. Acad. Sci. (USA). 1954. Vol. 40, no. 6. P. 471-474.

5. Архангельский А.В. Топологические пространства функций. М: Изд-во МГУ, 1989. 222 с.

6. Архангельский А.В. Строение и классификация топологических пространств и кардинальные инварианты // Успехи мат. наук. 1978. Т. 33, по. 6 (204). С. $29-84$.

7. Arhangel'skii A.V. Functional tightness, $q$-spaces and $\tau$-embeddings // Comment. Math. Univ. Carol. 1983. Vol. 24, no. 1. P. 105-120.

8. Окунев О.Г. О пространствах функций в топологии поточечной сходимости: расширение Хьюитта и $\tau$-непрерывные функции // Вестн. Моск. ун-та. Сер 1. Математика. Механика. 1985. № 4. C. $78-80$.

9. Куратовский К. Топология: в 2-х томах. Т.1. М.: Мир, 1966. 591 с.

10. Arhangel'skii A., Tkachenko M. Topological group and related structure. Paris: Atlantis Press; Hackensack, NJ: World Sci. Publ. Co. Pte. Ltd., 2008. 781 p. (Atlantis Studies Math.; vol. 1).

11. Stone A.H. Paracompactness and product spaces // Bull. Amer. Math. Soc. 1948. Vol. 54. P. 977-982.

12. Пестряков А.В. Бэровские функции и пространства бэровских функций: дис ... канд. физ.-мат. наук. Свердловск, 1987. 74 с.

Осипов Александр Владимирович

Поступила 3.06.2019

После доработки 12.08.2019

Принята к публикации 12.09.2019

д-р физ.-мат. наук, зав. сектором 
Институт математики и механики им. Н.Н. Красовского УрО РАН;

Уральский федеральный университет;

Уральский государственный экономический университет

г. Екатеринбург

e-mail: OAB@list.ru

\section{REFERENCES}

1. Hewitt E. Rings of real-valued continuous functions, I. Trans. Amer. Math. Soc., 1948, vol. 64, no. 1, pp. 45-99. doi: 10.2307/1990558.

2. Archangel'skii A.V., Ponomariov V.I. Fundamentals of General Topology: Problems and Exercises. N Y: Springer, 1984, 416 p. ISBN: 978-90-277-1355-1 . Original Russian text published in Arkhangel'skii A.V., Ponomarev V.I. Osnovy obshchei topologii v zadachakh i uprazhneniyakh. Moscow: Nauka Publ., 1974, $424 \mathrm{p}$.

3. Engelking R. General Topology. Sigma series in pure mathematics, vol. 6, Berlin: Heldermann Verlag, 1989, 535 p. ISBN: 3885380064 . Translated to Russian under the title Obshchaya topologiya, Moscow: Mir Publ., 1986, 752 p.

4. Nachbin L. Topological vector spaces of continuous functions. Proc. Nat. Acad. Sci. (USA), 1954, vol. 40, no. 6, pp. 471-474. doi: 10.1073/pnas.40.6.471.

5. Arkhangel'skii A.V. Topological function spaces. Math. its Appl., vol. 78, Dordrecht: Kluwer, 1992, 205 p. ISBN: 0-7923-1531-6. Original Russian text published in Arkhangel'skii A.V. Topologicheskie prostranstva funktsii, Moscow: MGU Publ., 1989, 222 p.

6. Arkhangel'skii A.V. Structure and classification of topological spaces and cardinal invariants. Russian Math. Surveys, 1978, vol. 33, no. 6, pp. 33-96. doi: 10.1070/RM1978v033n06ABEH003884.

7. Arhangel'skii A.V. Functional tightness, $q$-spaces and $\tau$-embeddings. Comment. Math. Univ. Carol., 1983, vol. 24, no. 1, pp. 105-120.

8. Okunev O.G. On function spaces in the topology of pointwise convergence: Hewitt extension and $\tau$ continuous functions. Vestn. Mosk. Univ., Ser. I, 1985, no. 4, pp. 78-80.

9. Kuratowski K. Topology. Vol. I. N Y; London: Acad. Press, 1966, 560 p. ISBN: 978-0-12-429201-7. Translated to Russian under the title Topologiya. T. 1, Moscow: Mir Publ., 1966, 594 p.

10. Arhangel'skii A., Tkachenko M. Topological group and related structure. Ser. Atlantis Studies in Math., vol. 1, Paris: Atlantis Press, 2008, 781 p. doi: 10.2991/978-94-91216-35-0.

11. Stone A.H. Paracompactness and product spaces. Bull. Amer. Math. Soc., 1948, vol. 54, pp. 977-982. doi: 10.1090/S0002-9904-1948-09118-2 .

12. Pestriakov A.V. Berovskie funktsii i prostranstva berovskikh funktsii (Baire functions and spaces of Baire functions). Cand. Sci. (Phys.-Math.) Dissertation. Sverdlovsk: Ural State of University Publ., 1987, 74 p.

Received June 3, 2019

Revised August 12, 2019

Accepted September 5, 2019

Alexander Vladimirovich Osipov, Dr. Phys.-Math. Sci., Krasovskii Institute of Mathematics and Mechanics of the Ural Branch of the Russian Academy of Sciences, Yekaterinburg, 620108 Russia; Ural Federal University, Yekaterinburg, 620083 Russia; Ural State University of Economicis, Yekaterinburg, 620144 Russia, e-mail: OAB@list.ru .

Cite this article as: A.V. Osipov. On the Hewitt realcompactification and $\tau$-placedness of function spaces, Trudy Instituta Matematiki i Mekhaniki URO RAN, 2019, vol. 25, no. 4, pp. 177-183. 